\title{
Metronomic Chemotherapy was Originally Designed and first used in 1994 for Early Stage Cancer - why is it Taking so Long to Proceed?
}

\section{Michael Retsky}

Harvard School of Public Health, 665 Huntington Avenue, Boston

\begin{abstract}
This is a personal case history from a researcher who was studying tumor growth when diagnosed in 1994 with stage IIIc colon cancer. The risk of relapse was $80 \%$ without therapy and $50 \%$ with conventional therapy. However his previous research led him to challenge the idea that tumor growth was described by the Gompertz equation. This is the fundamental theory underlying the concept that adjuvant chemotherapy should be started as soon as possible after primary surgery and should be administered at maximum tolerated dose and repeated when the patient's immune system recovers from the last course of therapy. It turns out that Gompertz growth is based on a study by Laird in the 1960s that consisted of measuring tumor growth on 18 rodents and one rabbit. On the basis of those data and a fundamental mathematical error, Laird claimed "The pattern of growth defined by the Gompertz equation appears to be a general biological characteristic of tumor growth." But then if the Gompertz equation assumption is wrong, how should adjuvant therapy be given? Patient/researcher opted for a low dose long term continuous infusion therapy with the mainstay colon cancer drug 5 -flourouracil. This therapy had previously been used in late stage disease but never in early stage disease. Patient used this non-toxic therapy 6 hours a night for 2.5 years. Patient was on the staff of Judah Folkman and after discussing this with Folkman, oncologist-researcher Tim Browder tested low dose long term continuous infusion 5- fluorouracil to determine if it is antiangiogenic. It was found to be so. This therapy is now called metronomic chemotherapy and is slowly being tested in laboratory, clinical and veterinary situations. Patient/researcher remains disease free 17 years later and asks why it is taking so long to proceed.
\end{abstract}

\section{Introduction}

When cancer is diagnosed in the breast or colon with no evidence of distant spread, the tumor is removed, the patient is given adjuvant therapy if there is sufficient concern for relapse, and then the patient is monitored for signs of metastatic relapse for a number of years thereafter. In the case of breast cancer such adjuvant therapy is hormone modulation if the primary tumor expresses estrogen or progesterone receptors or chemotherapy if it does not. There are no equivalent hormone receptors in colon cancer so therapy in that disease is always chemotherapy. The period of risk of relapse after therapy is of the order of 15-20 years in the case of breast cancer and 6-8 years for colon cancer.

Adjuvant chemotherapy is given approximately twice a month at or near maximum tolerated dosages and repeated when the patient's immune system recovers sufficiently. This is conducted for six or so months in order to decrease the probability that there will be a metastatic relapse - that is incurable and the common pathway to eventual demise from the disease. The underlying reason why chemotherapy is used that way is the belief that tumor growth is fastest when it is small and then gradually slows as the tumor gets larger. Therefore, since chemotherapy interferes with cell division, the best way to treat early stage cancer while minimizing collateral damage to normal cells is to do it intensively and to start as soon as possible after primary surgery. The theory is not much more complicated than that [1-5]. When these theories are discussed, the mathematical equation that is commonly cited to describe tumor growth is the damped exponential called Gompertzian. If you follow the chain of experimental evidence cited that tumors actually grow in a Gomptertzian manner, you always arrive at the 1960s papers by Laird [6,7].

In the US in 200841,000 persons died from breast cancer and 50,000 from colon cancer, most having had chemotherapy, so there is ample room for improvement. Something called drug resistance is blamed for eventual failure of chemotherapy. However, there is no clear molecular explanation why chemotherapy (or radiation for that matter) selectively kills tumor cells and why it ultimately fails [8].
I had been studying tumor growth since 1982 having made a career change from experimental physics to cancer biology. A person with my background is usually skilled in computational analysis and has much experience dealing with complex problems often with conflicting evidence. Those skills and that knowledge came in handy in November 1994 when, after a routine physical exam, I was diagnosed with stage IIIc colon cancer. There were 4 nodes positive and the (sigmoid) primary tumor had penetrated the muscularis propria and into the pericolonic fat. In the tumor, p53 was mutated and the tumor cells were aneuploid. After checking relevant papers I determined that my risk of metastatic relapse was $80 \%$ without any chemotherapy and about $50 \%$ with chemotherapy.

The primary tumor was removed (anastomosis) and recovery was uneventful. After speaking with a few medical oncologists, I decided against the standard six months of intensive adjuvant chemotherapy and instead opted for a low dose non toxic daily course using the commonly used drug 5-fluorouracil (5FU). The duration was not specified at the time but I ultimately stayed on this therapy for 2.5 years. This therapy that I chose as adjuvant therapy had been used occasionally in late stage disease but never before in early stage as I intended to use it.

Bear in mind that I am not a gambler. I have lived in Connecticut for over 20 years and have never gone to the casinos that are only an hour away. I purchased one lottery ticket in my life and that was for

*Corresponding author: Michael Retsky, PhD, Harvard School of Public Health, 665 Huntington Avenue, Boston, E-mail: michael.retsky@gmail.com

Received April 30, 2011; Accepted April 30, 2011; Published May 26, 2011

Citation: Retsky M (2011) Metronomic Chemotherapy was Originally Designed and first used in 1994 for Early Stage Cancer - why is it Taking so Long to Proceed? J Bioequiv Availab 3: 00i-0iv. doi:10.4172/jbb.100000e6

Copyright: (c) 2011 Retsky M. This is an open-access article distributed under the terms of the Creative Commons Attribution License, which permits unrestricted use, distribution, and reproduction in any medium, provided the original author and source are credited. 
a gag gift. What did I know in 1994 that so strongly convinced me to avoid the standard therapy for a life-threatening disease and instead take a different course that no one to my knowledge had ever used? In this paper I will discuss some of the evidence for Gompertzian kinetics, why I did not use short course and intensive adjuvant chemotherapy, and how this became known as metronomic chemotherapy.

\section{Discussion}

Gompertz was a 19th century actuarial scientist who proposed his equation as a general description of population growth. This growth starts exponentially (constant doubling time) and gradually slows down until it ultimately reaches a limiting plateau, as shown in Figure 1.

This is related to the Malthusian concept that the population of a city or state is ultimately limited by its food supply and ability to dispose of waste. Gompertzian growth is continuous, i.e., it cannot grow, stop, and then grow again. As mentioned, Gompertzian kinetics has played an important historical role in cancer chemotherapy and is still often cited. According to this theory, at the time of diagnosis of primary breast or colon cancer, metastatic disease is as small as it ever will be in the clinical setting-thus growing as fast and as chemosensitive as possible. Therefore, the optimal strategy for adjuvant chemotherapy is to use very intensive therapy as soon as possible after surgery for about 6 months and then hope for the best. This idea is traceable to the experimental work of Laird and by Skipper and Schabel in the 1960s and early 1970s [9-12].

Laird measured growth of "19 examples of 12 different tumors of the rat, mouse, and rabbit" and concluded: "The pattern of growth defined by the Gompertz equation appears to be a general biological characteristic of tumor growth." That is a far reaching statement based on only 18 rodents and one rabbit!

Laird compared a Gompertzian equation that was fit to growth data for each of the 19 animals. Then Laird graphed the exponential equation that has the same value and slope as the Gompertzian at time equal zero.

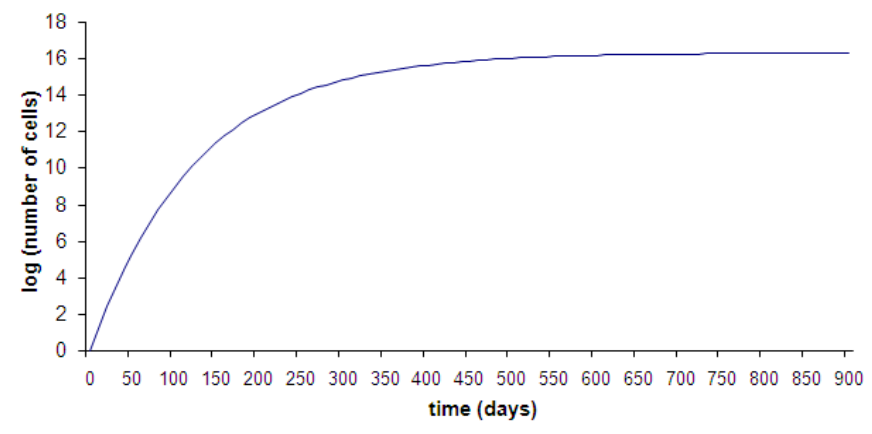

Figure 1: Gompertzian growth shown as log tumor burden (as represented by number of cells) vs. time (days). The equation is $N=\exp \left[(A / \alpha){ }^{*}(1-\exp [-\alpha t])\right]$ At small time the equation is exponential $(t A)$ and at large time, the expression approaches the asymptotic value of $\exp (\mathrm{A} / \mathrm{\alpha})$. The Gompertzian growth model has long been assumed to describe primary and metastatic tumors. The growth starts as exponential (constant doubling time) which would appear as a straight line on this semi-log scale. There are two parameters in this function that determine the initial growth rate and the ultimate size ( $N$ is the number of cells in the tumor). In figure $1, A$ was chosen as 0.3 per day and $\alpha$ was chosen to be 0.008 per day. Time $t$ is expressed in days. The ultimate size asymptotically attainable in this example is $1.9 \times 10^{16}$ cells. This value must be larger than $10^{12}$ cells, the value that is usually taken as a lethal tumor burden, since untreated cancer is presumed to be uniformly lethal. Thus, the two parameters $A$ and $a$ are not completely independent. $\operatorname{Exp}(A t)$ is equal to $\exp \left[(A / \alpha){ }^{*}(1-\exp [-\alpha t])\right.$ at $t=0$ but is larger at all other times greater than zero.

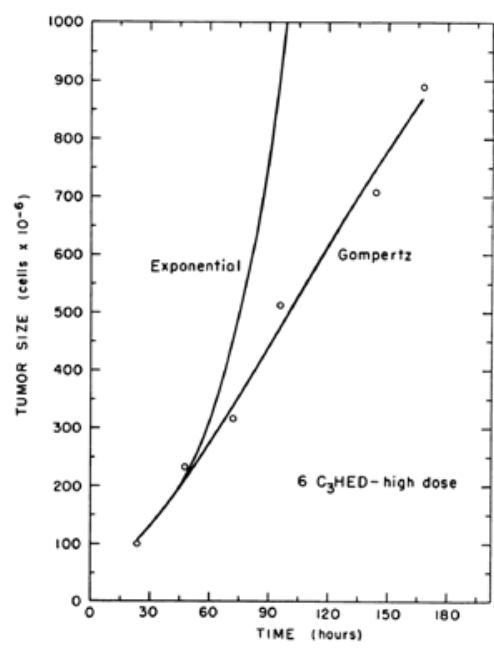

Figure 2: As modified from Laird [7], for one particular tumor Laird fit $W=W$ * $\exp [(0.788 / 0.142) *(1-\exp (-0.142 t))]$. That Gompertzian expression reasonably well fit those data. Then expanding $[1-\exp (-0.142 t)]$ in a Taylor series as $\left[1-1+0.142 t-0.142^{2} t^{2} / 2 !+\ldots\right]$ or $0.142 t$ (arbitrarily taking only the first non-zero term), Laird concluded that the exponential curve to compare to the best fit Gompertzian fit is $\exp [0.788 t]$. It is no surprise that the exponential starts at the same point as the Gompertzian but thereafter is always larger. This is a mathematical identity and has nothing to do with cancer.

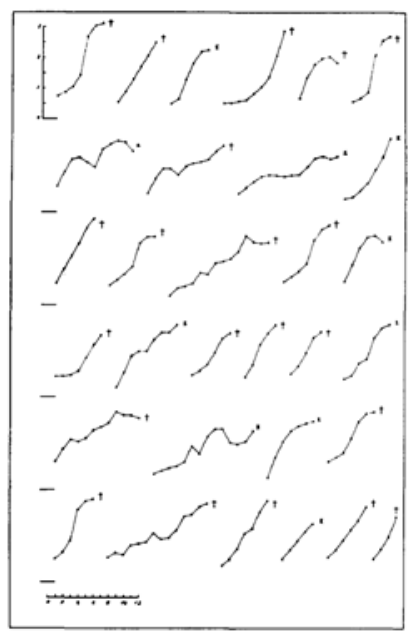

Figure 3: From Squartini [23] as modified. These are spontaneous tumors in animals that are genetically prone to cancer. That is, tumor cells are not injected nor are carcinogenic agents used to induce cancer. Horizontal scale as indicated is 12 weeks. Vertical scale as indicated is $3 \mathrm{~cm}$ diameter.

As no surprise, the pure exponential curve was accurate at $t=0$ but rapidly became far larger than the best fit Gompertzian. That was not a fair test to determine if Gompertzian kinetics or exponential kinetics best fit these data. This flawed analysis was conducted for each of the 19 tumors Laird reported. So in addition to using only 19 individual animals Laird used a flawed method to compare Gompertzian growth and exponential growth. Remarkably, Gompertzian kinetics as a valid description of breast cancer growth has been virtually unchallenged other than from my own publications starting in 1993 [13]. Laird papers have been cited over 600 times. Despite that, I came to the conclusion that I was the only person who actually read these papers and critically examined Laird's data. The main drawback in its use to design clinical 
treatments stems from the unsupported belief that it was explanatory of "all" the natural history of tumors in the subclinical phase and the clinical phase. It is also true that since a tumor consists of more than just cancer cells, the size of a tumor may not be an accurate measure of how many cancer cells are contained within.

Skipper and Schabel's work was done using multipassaged animal models with 1 or 2 day doubling times. They could cure animals if and only if all cancer cells were eradicated. Their papers included terms such as LD50 and LD90 to indicate that some of their chemotherapy protocols were lethally toxic to $50 \%$ or $90 \%$ of animals. The high success of using chemotherapy at maximum tolerate dose to treat animal model tumors has translated into only modest benefit in clinical breast and colon cancer and accompanied by significant toxicity. Skipper and Schabel considered human tumor growth to be Gompertzian and cited Laird as evidence. Their recommended method of treatment was to start as soon as possible using maximum tolerated doses repeated as soon as the host's immune system recovered sufficiently from the previous treatment. The Skipper and Schabel papers have been cited over 2000 times and were very influential in oncology.

Additional information available to me before being diagnosed with colon cancer are data I published in 1990 [14]. I was Visiting Professor at University of Texas Health Science Center at Dr. William McGuire's invitation. I was working on a method to include hormone receptors into a computer simulation of breast cancer that I developed previously. For about 6 months, I was spending alternate weeks in San Antonio while my family was living in Colorado Springs. In San Antonio, I was given a few columns from their 57,000 patient database but, as we found out months later, one of the columns provided to me was mislabeled. Instead of what I was told was "date of relapse" it was actually "date of last examination of patient". As a result of the mislabeling, I could not make any progress for a number of months. Since my research was not getting anywhere, I had time to do other things and spent much time in their well stocked medical library. I was looking for serial data on tumor growth in untreated conditions. In the older literature "watchful waiting" was a common practice which is quite different from what happens today when a tumor is identified.

The Briscoe Medical Library had an extensive collection of older journals. If I was reading one paper and saw some interesting references I could go to the stacks and examine all the referenced journals. This rarely happens in libraries when researching in the journals from decades past. As a result I was able to identify over 100 examples of tumor growth in animals and humans that showed how naturally occurring untreated tumors increased in size with time. I am quite competent in fitting algebraic functions to data but it would be sheer folly to try and come up with an equation that even resembles general tumor growth. Untreated tumors tend to increase in size over time but with no particular mathematically description that can remotely be said to cover more than a few tumors of the over 100 shown in Figure 2 (As we now know tumor growth especially for breast cancer is very complicated [15]. One of the figures showing serial data on growth for 30 spontaneous animal tumors from this 1990 paper is shown as Figure 3

By 1994 I was sure that there was a big difference in growth between multipassaged animal tumors as used by Laird and by Skipper and Schabel and spontaneous cancer and I especially questioned the reliance on Gompertzian kinetics to guide therapy.

When I was diagnosed at the end of October 1994, it can truly be said that I seriously doubted the dogma that adjuvant chemotherapy should be maximum tolerated dosage given intermittently for 6 months or so and then hope for the best. I was very dissolutioned with the science behind a therapy that had been and still is administered to a great many breast and colon cancer patients.

As mentioned, I used low dose long term therapy with the standard colon cancer drug for 2.5 years. It was administered for 6 hours per night using a portable pump through a double lumen port implanted on my chest under the skin. In the morning I disconnected the pump and flushed the thin plastic tubes. At bedtime I reprogrammed the pump and connected it back to the tubes. The original protocol was designed by medical oncologist Dr. William Hrushesky who had used this therapy on late stage patients but never before in early stage. (As originally designed, therapy was circadian rhythm timed but I gradually changed to a more convenient bedtime schedule.) The dosage chosen was $30 \%$ below the long term tolerable limit of $300 \mathrm{mg} / \mathrm{m}^{2} /$ day and since the toxicity profile is very steep, there was virtually no toxicity. I knew that I was getting the drug since there were small cracks in the skin at my finger joints, the finger tips were soft and somewhat numb and my fingernails grew coarsely. I had a few blood blisters inside my mouth. That was the full extent of the toxicity.

Over that period I did not miss a day of work, took no pain medicine, did not vomit and felt well enough to exercise vigorously daily. (I did not have much hair to start with so can't say much about hair loss.) I was doing cancer research at Harvard Medical School during most of that period and on the staff of the late Judah Folkman. As many know, Dr. Folkman founded the field of tumor angiogenesis and was one of the most influential persons in the world in cancer research. He knew of my therapy and encouraged me to continue.

I was carefully monitored. In addition to the usual imaging studies, I had two conventional and two experimental tumor markers measured every month for over 5 years. There was one time when two of the markers temporarily rose slightly from a steady low level but never exceeded "normal" levels. Other than that, all markers were low and steady.

While in therapy, I discussed my treatment with Dr. Folkman. I knew that in the Folkman lab they were testing many compounds and substances for pro and antiangiogenic activity. I also knew from Dr. Hrushesky and other sources that low dose infusional 5FU apparently worked for extended periods of time seemingly without developing drug resistance. At that time the understanding was that an antiangiogenic therapy would not develop drug resistance. I therefore suspected that low dose infusional 5FU as I was using it might have antiangiogenic properties in addition to cytotoxic activity. I asked Dr. Folkman if they tested 5FU as bolus treatment as conventionally used or as long term low dose as I used it. Apparently it was only tested as bolus (high dose) and it was not antiangiogenic. Dr. Folkman then brought Dr. Tim Browder into the room. Browder was a pediatric medical oncologist and was the person conducting those measurements in the laboratory. It was clear that they had not tested it as I used it. Browder later purchased some micro implantable pumps and eventually tested 5FU and a number of other common cancer drugs at low dose for extended periods of time in mice and found that several of them including $5 \mathrm{FU}$ were antiangiogenic if administered that way. It took several years for Browder to get his findings published since they were totally unexpected. Finally they were published in 2000 [16].

I did not relapse and am now well beyond the period of risk of relapse for colon cancer so am $80 \%$ sure the therapy worked as planned for me. 
Citation: Retsky M (2011) Metronomic Chemotherapy was Originally Designed and first used in 1994 for Early Stage Cancer - why is it Taking so Long to Proceed? J Bioequiv Availab 3: 00i-Oiv. doi:10.4172/jbb.100000e6

\section{Conclusions}

Robert Cooke happened to be writing an authorized biography of Judah Folkman and at Folkman's direction interviewed me in 1999. By that time I was fairly confident the therapy worked. Either that or surgery was curative and the therapy was unnecessary. By that time, I had told many patients and physicians about low dose long term 5FU since Dr. Folkman directed them to me. I was also active as a patientadvocate and a founder of the Colon Cancer Alliance. (I am still on the Board of Directors.) Cooke's biography of Judah Folkman was published in 2000 and there are three pages that discuss my medical history [17]

Unfortunately both Judah Folkman and Tim Browder are deceased. Dr. Folkman had introduced me on several occasions as the first human to use (adjuvant) metronomic chemotherapy - as this therapy is now known. The Browder et al paper has been cited over 600 times and metronomic chemotherapy has been tested many times especially in late stage disease. However I am sad to report that it is now 17 years since I used it and metronomic chemotherapy has still not been fully tested for use as originally designed in adjuvant treatment for early stage breast or colon cancer [18-21]. At this rate it could be another 17 years before it is fully tested and then commonly used - assuming it works as well for others as it did for me. I had been unable to stimulate much interest in testing my therapy and a major reason is that $5 \mathrm{FU}$ is less expensive than sterile water. Who wants to spend a lot of time and money promoting a therapy when there is no financial benefit if it works? Maybe the research community can find an easier way to accomplish this with a different drug that is even more effective and that does not need to be infused over 6 hours daily. Perhaps Xeloda, the oral pro-drug for $5 \mathrm{FU}$ can be used instead. Hopefully my writing this paper will help speed the way toward the testing and usage of a nontoxic low dose chemotherapy for early stage cancer. As a final comment, the elementary mathematical flaw in Laird's papers and the fact that 600 cancer researchers cited that study suggests that cancer researchers might consider additional training in basic mathematics.

\section{Acknowledgement}

Michael Retsky is supported by grant KG 100484 from Komen Foundation Disclosure - patent pending on method to treat early stage breast cancer

\section{References}

1. Cooper MR (1991) Principles of medical oncology. In: Holleb Al, Fink DJ Murphy GP, editor. In American Cancer Society Textbook of Clinical Oncology. Atlanta: American Cancer Society 47-68.

2. Hellman S, DeVita VT Jr. (1982) Principles of cancer biology: kinetics of cellular proliferation. In: DeVita Jr, Hellman S, Rosenberg S, editor. In Cancer: Principles and Practices of Oncology 13.

3. Norton L (1988) A Gompertzian model of human breast cancer growth. Cancer Res 48:7067-7071.

4. Retsky M, Swartzendruber D, Wardwell R, Bame P, Petrosky V (1989)
Correspondence re: Larry Norton. A Gompertzian Model of Human Breast Cancer Growth. Cancer Res 49: 6443-6444.

5. Prehn RT (1991) The inhibition of tumor growth by tumor mass. Cancer Res 51: $2-4$.

6. Laird AK (1964) Dynamics of tumor growth. Br J Cancer 13: 490-502.

7. Laird AK(1969) Dynamics of growth in tumors and in normal organisms. Nat Cancer Inst Monogr 30: 15-28.

8. Luo J, Solimini NL, Elledge SJ (2009) Principles of cancer therapy: oncogene and non-oncogene addiction. Cell 136: 823-837.

9. Skipper HE, Schabel FM Jr, Trader MW, Laster WR Jr, Simpson-Herren L, et al. (1970) Basic and therapeutic trial results obtained in the spontaneous AK leukemia (lymphoma) model-end of 1971. Cancer Chemother Rep 56: 273287.

10. Skipper HE, Schabel FM Jr, Mellett LB, Montgomery JA, Wilkoff LJ, et al. (1970) Implications of biochemical, cytokinetic, pharmacologic, and toxicologic relationships in the design of optimal therapeutic schedules. Cancer Chemothe Rep 54: 431-50.

11. Skipper HE, Schabel FM Jr, Wilcox WS (1964) Experimental Evaluation of Potential Anticancer Agents. XIII. On the Criteria and Kinetics Associated with Curability of Experimental Leukemias. Cancer Chemotherapy Rept 85: 1-111.

12. Band PR (2010) The birth of the subspecialty of medical oncology and examples of its early scientific foundations. J Clin Oncol 28: 3653-3658.

13. Retsky MW, Swartzendruber DE, Wardwell RH, Bame PD (1993). A new paradigm for breast cancer. Recent Results Cancer Res 127: 13-22.

14. Retsky M, Swartzendruber D, Wardwell R., Bame P (1990) Is Gompertzian Kinetics a Valid Description of Individual Tumor Growth? Medical Hypothesis 33: 95-106.

15. Retsky M, Demicheli R, Hrushesky W, Baum M, Gukas I (2010) Surgery Triggers Outgrowth of Latent Distant Disease in Breast Cancer: An Inconvenient Truth? Cancers 2: 305-337.

16. Browder T, Butterfield CE, Kräling BM, Shi B, O'Reilly MS, et al. (2000) Antiangiogenic scheduling of chemotherapy improves efficacy agains experimental drug-resistant cancer. Cancer Res 60: 1878-1886.

17. Cooke R (2000) Dr Folkman's War: Angiogenesis and the Struggle to Defeat Cancer. New York: Random House 328-350.

18. Calvani N, Orlando L, Nacci A, Sponziello F, Cinefra M, et al.(2009) Metronomic chemotherapy against cancer: from paradigm to clinical practice? Tumori 95 843-845.

19. Brandi G, de Rosa F, Bolondi L, Agostini V, Di Girolamo S, et al. (2010) Durable complete response of hepatocellular carcinoma after metronomic capecitabine. Tumori 96: 1028-1030.

20. Mutsaers AJ (2009) Metronomic chemotherapy. Top Companion Anim Med 24 137-143.

21. Emmenegger U, Kerbel RS (2007) Five years of clinical experience with metronomic chemotherapy: achievements and perspectives. Onkologie 30 606-608.

22. Squartini $F(1961)$ Strain differences in growth on mouse mammary tumors. J Nat. Cancer Inst 26: 813

23. Retsky M, Swartzendruber D, Wardwell R, Bame P (1994) Computer mode challenges breast cancer treatment strategy. Cancer Investigation 12: 559-567. 